Proceedings

\title{
A Novel Low Cost Wireless Incontinence Sensor System (Screen-Printed Flexible Sensor System) for Wireless Urine Detection in Incontinence Materials ${ }^{+}$
}

\author{
Ha-Duong Ngo 1,2,*, Thanh Hai Hoang ${ }^{1}$, Manuel Baeuscher 1,3,*, Bei Wang ${ }^{2}$, Piotr Mackowiak ${ }^{1}$, \\ Nils Grabbert 1, Thomas Weiland 1, Oswin Ehrmann 1,3, Klaus-Dieter Lang 1,3, \\ Martin Schneider-Ramelow ${ }^{1,3}$ and Jens Grudno ${ }^{4}$ \\ 1 Fraunhofer Institute IZM, Gustave-Meyer-Allee 25, 13355 Berlin, Germany; \\ thanh-hai.hoang@izm.fraunhofer.de (T.H.H.); piotr.mackowiak@izm.fraunhofer.de (P.M.); \\ niels.grabbert@izm.fraunhofer.de (N.G.); thomas.weiland@izm.fraunhofer.de (T.W.); \\ oswin.ehrmann@izm.fraunhofer.de (O.E.); klaus-dieter.lang@izm.fraunhofer.de (K.-D.L.); \\ martin.schneider-ramelow@izm.fraunhofer.de (M.S.-R.) \\ 2 University of Applied Sciences Berlin, Wilhelminenhofstr. 75A, 12459 Berlin, Germany; \\ bei.wang@htw-berlin.de \\ 3 Technical University Berlin, Centre of Microperipheric Technologies, Gustav-Meyer-Allee 25, \\ 13355 Berlin, Germany \\ 4 Assistr Digital Health Systems GmbH, Lohmühlenstraße 65, 12435 Berlin, Germany; \\ jens.grudno@assistrcare.com \\ * Correspondence: ha-duong.ngo@izm.fraunhofer.de (H.-D.N.); manuel.baeuscher@izm.fraunhofer.de (M.B.) \\ † Presented at the Eurosensors 2018 Conference, Graz, Austria, 9-12 September 2018.
}

Published: 21 December 2018

\section{Summary}

In this paper we present a printed, fast, high-sensitive and cheap wireless incontinence sensor system, which could be integrated in a common diaper for urinary detection. The system consists of a flexible screen-printed IDEs (Inter Digital Electrodes) sensor with bio compatible conducting PEDOT:PSS material, a battery, a readout electronics for sensor signal and a wireless module.

\section{Introduction and Motivation}

Today's sensors and microelectronics are bulky and rigid since they are made in semiconductors such as silicon. Unlike conventional microelectronics/nanoelectronics, next-generation sensors and electronics focus on large-area and low-cost applications and new form factors such as flexible and stretchable devices [1,2]. Electronic devices that are fabricated on plastic or rubbery substrates such as flexible display, electronic paper, smart packages, skin-like sensors, wearable electronics, medical electronic implants, and many others, could radically change people's perspectives on electronics [2].

Urinary incontinence-the loss of bladder control-is a significant health problem with considerable social and economic impact on individuals and society [3]. It occurs more often as people get older. A number of factors are contributing to the growth of the adult incontinence market [4]. According the U.S. National Institute of Health, about 20 million adult women and six million adult men experience or have experienced urinary incontinence in the U.S. and there are countless more living with fecal incontinence. Incontinence does not alone affect older people. In fact, men, women and children of all ages are living with the condition, but it is more common by older adults, particularly women who have had children [5]. Using diaper with absorber material as one-way product is the most used method against incontinence. The adult diapers market is huge. According 
to the Zion Market Research the adult diaper market was valued at USD 9246.36 million in 2015 and is expected to reach USD 14,592.72 million by 2021, growing at a CAGR of 7.5\% between 2016 and 2021. In term of volume, the global adult diapers market stood at 17,689.30 million units in 2015 [6].

In many cases, the amount of moisture in the diaper is checked "manually" by the nursing staff in a certain time period. This care procedure is annoying for both, patients and the staff. The idea in this work is to develop a smart cheap one-way sensor platform integrated in diaper, which is able to detect the moisture and serves as sensor platform to detect chemical properties or bio-markers in urine in future.

\section{Sensor design and manufacturing}

Main important requirements for the sensor systems could be seen below.

- detect the amount of the liquids inside the diaper and gives electrical signal to cloud or caregiver

- low cost

- $\quad$ bio compatible (ISO 10993)

- flexible and stretchable

- $\quad$ easy integration in a standard diaper

- $\quad$ wireless interface compatible to standards in medical systems.

Among the many physical principles (for example optical, chemical and electrical), which is suitable to measure the humidity, the resistive and capacitive methods do have many advantages, such as easy manufacturing, standard electronics for data acquisition. The resistive and capacitive structures could be integrated directly on flexible substrates. Figure 1 below shows the sensor concept. The single sensor is containing planar inter digital electrodes (IDEs) between the diaper foil and the absorbent pad. The absorbed urine on top of IDEs changes the sensor resistance and capacitance. The sensor array consists of 6 sensors, and is able to detect the urine amount in the diaper, and sends the signal to cloud or to nurse or caregivers.

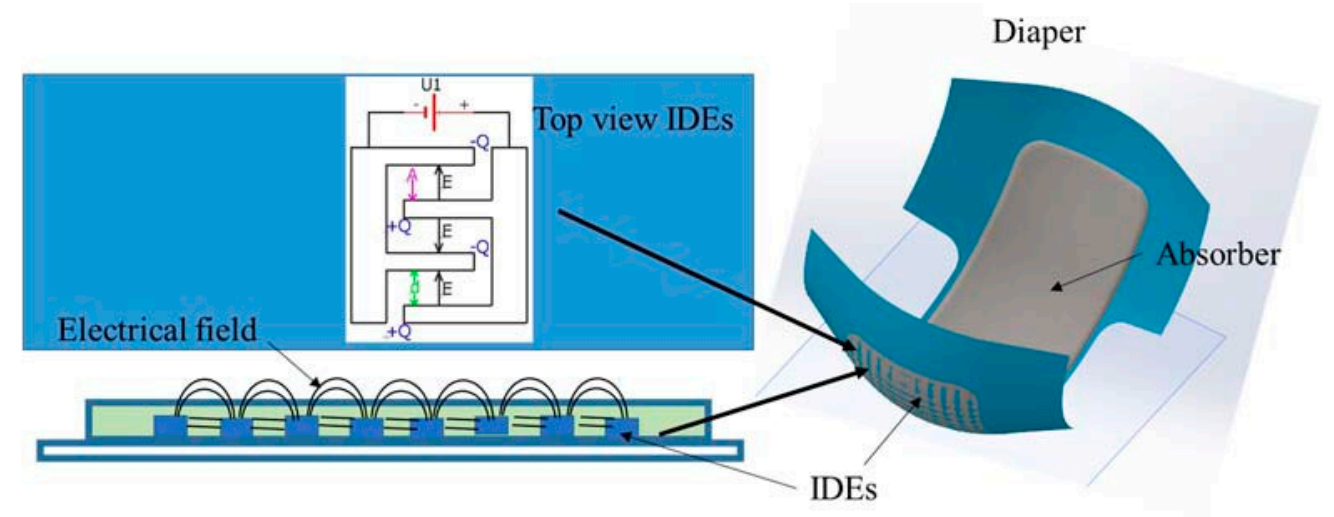

Figure 1. Sensor concept for detection of urine in incontinence materials. The sensor system is consisting of distributed single sensors integrated into diaper. The resistance and the capacitance of the sensor is depending on the amount of urine.

Common resistive and capacitive humidity sensors have been fabricated in silicon. But as mentioned, silicon technology has some limitations, such as low flexibility, low stretchability and high invest. Another limitation is a high number of silicon humidity sensors, which would be necessary in one diaper. In this work PEDOT:PSS (AGFA ORGACON EL-P5015, viscosity >100 Pa·s) conducting polymer [7] was used as material to form the IDEs structures. The material is highly flexible and has a sheet resistivity of $125 \Omega / \square$. The real foil substrate has a size of $280 \times 660 \mathrm{~mm}^{2}$. The process flow is shown in Figure 2. The IDEs are printed on the foil, cured by $80{ }^{\circ} \mathrm{C}$ for $30 \mathrm{~min}$, and laminated on paid material. 


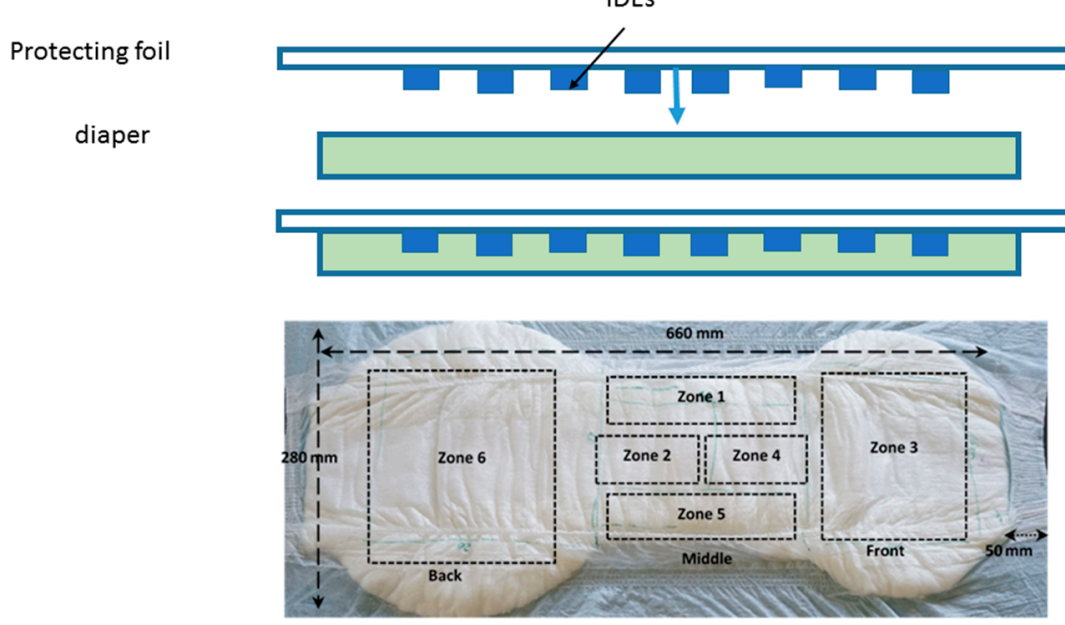

Figure 2. Integration of the novel smart incontinence sensor into standard diaper. First IDE array is realized on protecting foil by using screen printing. In the next step the foil is laminated on the bottom of the diaper. Foil and lamination are standard material and standard procedure in diaper production.

The geometries of the 6 sensors are shown in the Table 1 below.

The system uses RF Digital RFD77101 Simblee ${ }^{\mathrm{TM}}$ Bluetooth ${ }^{\circledR}$ Low Energy for data acquisition and signal transmission. With a standard battery (CR2032) the system has a life time of about 6 months before the battery has to be changed.

Table 1. Sensor geometry, finger width $2 \mathrm{~mm}$, distance $2 \mathrm{~mm}$, thickness $1 \mu \mathrm{m}$.

\begin{tabular}{ccc}
\hline Sensor & Size L $(\mathbf{m m})$ & Number of the Sensor's Fingers $\mathbf{N}$ \\
\hline 1 & 38 & 46 \\
2 & 88 & 12 \\
3 & 158 & 38 \\
4 & 38 & 46 \\
5 & 88 & 12 \\
6 & 208 & 38 \\
\hline
\end{tabular}

\section{Results and Conclusions}

The printed sensor array is shown in Figure 3 . The foil size is $70 \times 32 \mathrm{~cm}^{2}$.

It is perfectly suitable for wearable and one-way products. Test has been made to test the developed system with synthetic urine (Human-Urin-Imitat 1000-D). Figure 4 shows the prototype. There is no issues to be observed in term of flexibility and stretchability in comparison with standard products on the market. Figure 5 shows the sensors signals as function of urine amount in the diaper.

The new developed system has shown very good and repeatable performances. Test is still ongoing to improve mechanical and chemical stability in extreme conditions. Development work is still necessary to scale up the process for mass production. Capacitive principle has many advantages in compare to resistive one, such as the possibility of using a thin flexible passivation layer to protect the PEDOT:PSS structures.

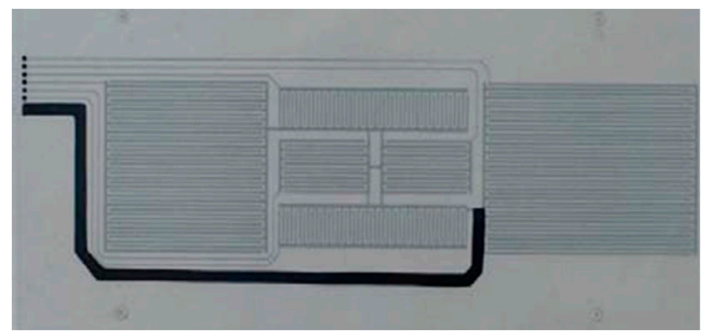

Figure 3. A printed sensor array with 6 sensors. PEDOT:PSS-IDEs on diaper foil. 


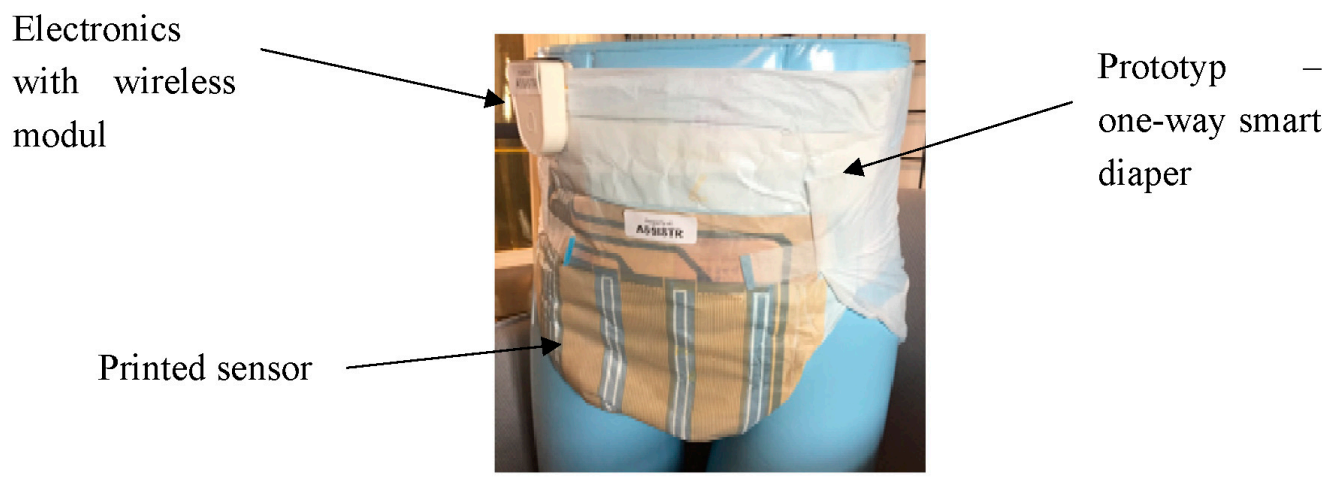

Figure 4. The smart diaper prototype with electronics and wireless module.

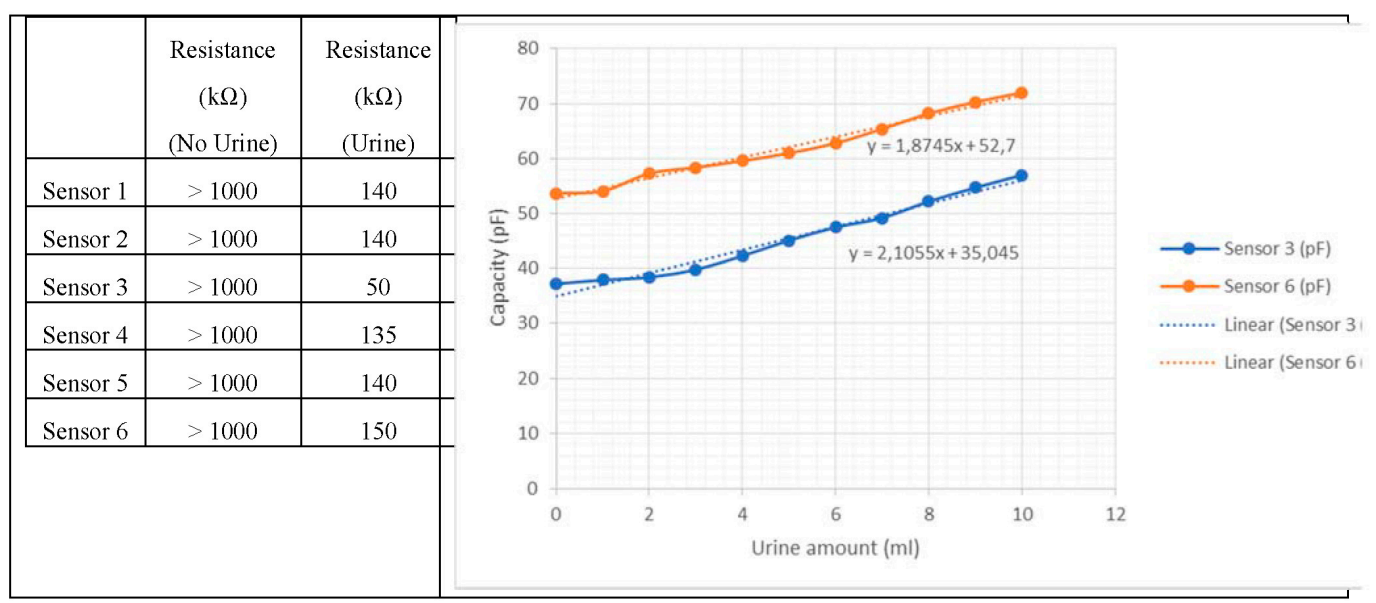

Figure 5. Sensor signals as function of urine. Synthetic urine was used. Left-resistance signal, and right-capacitive signal.

\section{References}

1. Someya, T. Stretchable Electronics; Wiley-VCH: Weinheim, Germany, 2013; ISBN 978-3-527-32978-6.

2. Takei, K. Flexible and Stretchable Medical Devices; Wiley-VCH: Weinheim, Germany, 2018; ISBN 978-3-52734183-2.

3. Nitti, V.W. The prevalence of urinary incontinence. Rev. Urol. 2001, 3, S2-S6.

4. Olivo, T. Adult Incontinence: A Market Rife with Opportunity, 2017. Available online: https://www.nonwovens-industry.com/issues/2017-03-01/view_features/adult-incontinence-a-marketrife-with-opportunity (accessed on 20 December 2018).

5. National Institute of Mental Health. Mental Illness; National Institute of Mental Health: Bethesda, MD, USA, 2017.

6. Zion Diapers Market (Pad Type, Pants Type, and Flat Type Adult Diapers): Global Industry Perspective, Comprehensive Analysis, and Forecast, 2015-2021. Available online: https://www.zionmarketresearch.com/news/global-adult-diapers-market (accessed on 20 December 2018).

7. Magdassi, S. Nanomaterials for 2D and 3D Printing; Wiley-VCH: Weinheim, Germany, 2017; ISBN 978-3-52733819-1.

(C) 2018 by the authors. Licensee MDPI, Basel, Switzerland. This article is an open access article distributed under the terms and conditions of the Creative Commons Attribution (CC BY) license (http://creativecommons.org/licenses/by/4.0/). 